trois ans de séropositivité, $10 \%$ après quatre ans, $15 \%$ après cinq ans, $24 \%$ après six ans et $36 \%$ après sept ans [3].

L'infection par HIV-2 est surtout fréquente en Afrique de l'Ouest et s'étend rapidement. Il est sûr maintenant que HIV -2 est pathogène, mais la fréquence d'apparition des signes cliniques chez les séropositifs reste inconnue.

Les principales mesures prophylactiques susceptibles de diminuer les risques d'infection sont l'utilisation constante de préservatifs masculins (qui ne donne cependant une protection que de $90 \%$ par contact sexuel et environ 62 à $70 \%$ pour une probabilité cumulée d'être infecté après 1000 contacts sexuels) et la réduction du nombre de partenaires sexuels. Encore faut-il, pour qu'elle soit « efficace», que cette réduction soit importante, puisque le risque diminue peu pour un individu passant de 50 à cinq partenaires sexuels sur quelques années, le «bénéfice » devenant important pour une réduction à un partenaire exclusif [2].
A. K.
1. Piot P, Plummer FA, Mhalu FS, Lamboray JL, Chin J, Mann JM. AIDS : an international perspective. Science $1988 ; 239$ : $573-9$.

2. Fineberg HV. Education to prevent AIDS : prospects, obstacles. Science $1988 ; 239: 592-6$.

3. Curran JW, Jaffe HW, Hardy AM, Morgan WM, Selik RM, Dondero TJ. Epidemiology of HIV infection and AIDS in the United States. Science 1988; 239: 610-6.

\section{Pourquoi l'intoxication par la réglisse entraîne-t-elle une hypertension artérielle?}

L'activité gluco- et minéralocorticoïde des stéroïdes est liée à l'existence d'un groupe hydroxyle en Cll. Le cortisol actif est transformé habituellement en cortisone inactive grâce à un complexe enzymatique microsomial, principalement hépatique et rénal, la $11 \beta$ hydroxystéroïde déshydrogénase ( $11 \beta \mathrm{OH}$ $\mathrm{SD})$. Le déficit en cette enzyme aboutit à des concentrations intratissulaires importantes de cortisol, qui agit alors comme un puissant minéralocorticoïde, et à une augmentation d'excrétion urinaire des métabolites du cortisol (aux dépens de celle des métabolites de la cortisone), alors que la cortisolémie reste normale.

Le syndrome d'excès apparent de minéralocorticoïdes est un syndrome rare, responsable chez l'enfant d'une hypertension artérielle avec hypokaliémie et inhibition du système rénine-angiotensine-aldostérone. Il est secondaire à un déficit congénital en $11 \beta \mathrm{OH}-\mathrm{SD}$. Cela augmente le rapport entre les métabolites urinaires du cortisol et ceux de la cortisone. Ce syndrome est corrigé par la dexaméthasone qui supprime la production de cortisol.

L'intoxication par la réglisse entraîne une hypertension artérielle, une hypokaliémie et une inhibition du système rénine-aldostérone. Depuis longtemps, ces anomalies ont été attribuées à l'effet minéralocorticoïde faible de l'acide glycyrrhizinique (provenant de la réglisse). Récemment Stewart et al. [1] ont remarqué l'analogie entre l'intoxication par la réglisse et le syndrome d'excès apparent de minéralocorticoïdes : les manifestations cliniques sont superposables ; dans les deux cas, les antagonistes de l'aldostérone sont efficaces ; dans l'intoxication par la réglisse, la dexaméthasone corrige également les anomalies et celles-ci ne se développent pas chez le sujet insuffisant surrénalien. Ces auteurs ont donc étudié les effets de la prise d'acide glycyrrhizinique pendant dix jours chez sept hommes volontaires sains. Outre les manifestations attendues, ils ont observé une augmentation du cortisol libre urinaire, sans modification du cortisol plasmatique, et une augmentation du rapport métabolites urinaires du cortisol/métabolites urinaires de la cortisone. Ces modifications surviennent pour des concentrations plasmatiques d'acide glycyrrhizinique inférieures à celles nécessaires pour déplacer l'aldostérone de son récepteur - c'est-à-dire pour exercer une activité minéralocorticoïde.

Les conséquences cliniques de l'intoxication par la réglisse pourraient donc bien relever de l'inhibition de la $11 \beta \mathrm{OH}-\mathrm{SD}$, à l'image de ce qu'on observe dans le déficit congénital de cette enzyme.

G. B.

\section{BRÈVES}

L'ichthyose congénitale liée à l'X semble presque constamment secondaire à une délétion du gène de la stéroïde-sulfatase. Cette maladie est due à l'absence de l'enzyme qui dégrade normalement le cholestérolsulfate dont l'abondance dans la peau inhibe la desquamation de la couche cornée, conduisant ainsi à son épaississement caractéristique de l'ich thyose. Dans 40 des 45 familles étudiées jusqu'à présent par différents auteurs [1], la base moléculaire de la maladie est une délétion du gène L'ichthyose congénitale semble à l'heure actuelle l'une des affections dans laquelle la plus haute proportion de mécanismes délétionnels a été observée. Cette particularité s'ajoute aux autres «bizarreries » du gène de la stéroïde-sulfatase : situé à l'extrémité du bras court, il est très proche de la région à transmission pseudoautosomique chez l'homme... et dans cette région chez la souris $(\mathrm{m} / \mathrm{ssuppl}$. au $n^{\circ} 7$, vol. 3, p.11). De plus, il échappe partiellement à l'inactivation au hasard d'un chromosome $\mathrm{X}$ au cours de l'embryogenèse.

[1. Bonifas JM, et al. Proc Natl Acad Sci USA 1987 ; 84 : 9248-51]

1. Stewart PM, Valentino $R$, Wallace AM, Burt D, Shackleton CHL, Edwards CRW. Mineralocorticoid activity of liquorice: 11. beta-hydroxysteroid dehydrogenase deficiency comes of age. Lancet $1987 ; 2: 821-3$. 